\title{
NOTE
}

\section{Kinetic Analysis of Engineered Polyhydroxyalkanoate Synthases with Broad Substrate Specificity}

\author{
By Ken'ichiro MATSUMoto, ${ }^{1,2, *}$ Fumi SHOZUI, ${ }^{1}$ Yasuharu SATOH, ${ }^{1}$ \\ Kenji TAJIMA, ${ }^{1}$ Masanobu MUNEKATA,${ }^{1}$ and Seiichi TAGUCHI ${ }^{1}$
}

KEY WORDS: Polyhydroxyalkanoate / PHA Synthase / Kinetic Analysis / Pseudomonas Sp. 61-3 /

Polyhydroxyalkanoates (PHAs) are bacterial polyesters that can be processed to useful thermoplastic materials, such as films and fibers, thus expected to be an alternative to petrochemical plastics. ${ }^{1}$ The polyesters are produced from renewable carbon source and well-biodegradable. ${ }^{2}$ These environmentally friendly features of PHAs have attracted the research interest to produce PHA for commercial use.

PHAs are typically synthesized by $R$-form specific polymerization of 3-hydroxyacyl-coenzyme A (3HA-CoA) with various carbon lengths, which is catalyzed by PHA synthase. ${ }^{3-5}$ The substrate specificity of PHA synthase is a dominant factor determining the monomer composition of PHA, which affects thermal and physical properties of the material. In particular, PHA copolymers consisting of short-chain-length (SCL, $\mathrm{C}_{4}$ and $\mathrm{C}_{5}$ ) monomers and medium-chain-length (MCL, $\mathrm{C}_{6}$ to $\mathrm{C}_{14}$ ) monomers (SCL/MCL PHA) are tough and flexible material, and have wide range of applications. The properties of the copolymers are largely altered depending on the ratio of SCL and MCL monomers. Thus, regulation of monomer composition is very important to produce PHA with desirable properties. $^{6-9}$

PHA synthase from Pseudomonas sp. 61-3, which belongs to class II, has especially broad substrate specificity and synthesizes SCL/MCL PHA copolymer. ${ }^{10}$ However, weak activity toward SCL monomer has limited productivity of SCL/MCL PHAs and regulation of their monomer composition. Therefore, we previously created various mutants of the PHA synthase to enhance the incorporation activity of SCL unit. ${ }^{11,12}$ Through enzyme evolutionary engineering, ${ }^{13}$ we obtained several beneficial mutants, which exhibited SCL PHA, poly(3-hydroxybutyrate) $[\mathrm{P}(3 \mathrm{HB})]$, content (up to $40 \mathrm{wt} \%)$ in recombinant Escherichia coli compared to the wild-type PHA synthase $(0.1 \mathrm{wt} \%)$. The selected mutants were expected to have enhanced activity toward SCL monomer. Indeed, they increased productivity and altered monomer composition of the SCL/MCL PHA copolymer produced in E. coli, ${ }^{5,14-16}$ suggesting that these beneficial mutations have altered activity toward SCL and MCL monomers. The detailed characterization of mutational effect on the activity of PHA synthase is necessary for selecting proper mutants and also further engineering of the enzyme. Therefore, we determined the kinetic parameters of wild-type and mutated PHA synthases to clarify the effects of beneficial mutations and their combinations.

\section{EXPERIMENTAL}

\section{Plasmid Construction}

The wild-type PHA synthase gene from Pseudomonas sp. $61-3^{10}$ was amplified using following primers: 5'-CCGGATCCAGTAACAAGAATAGCGATGACTTGA-3' and 5'-TTTAAGCTTAACGTTCATGCACATACGTG-3'. The restriction sites used for gene construction were underlined. The BamHI/HindIII fragment of PHA synthase gene was inserted into pQE30 (QIAGEN) to yield pQEC1WT, which encodes the His-tag fusion of PHA synthase. The pQEC1 plasmids harboring mutated PHA synthase genes, pQEC1ED (E130D mutant), pQEC1ST (S325T), pQEC1QK (Q481K), pQEC1EDST (E130D/S325T), pQEC1EDQK (E130D/ Q481K), pQEC1STQK (S325T/Q481K), and pQEC1EDSTQK (E130D/S325T/Q481K), were constructed by recombining the ClaI/BglII fragments of PHA synthase gene.

\section{Medium and Buffers}

Terrific broth contains $12 \mathrm{~g} / \mathrm{L}$ bacto-trypton, $24 \mathrm{~g} / \mathrm{L}$ bactoyeast extract, $4 \mathrm{~mL}$ glycerol, $0.17 \mathrm{M} \mathrm{KH}_{2} \mathrm{PO}_{4}$, and $0.72 \mathrm{M}$ $\mathrm{K}_{2} \mathrm{HPO}_{4}$. Four buffers were used for enzyme purification as follows: $50 \mathrm{mM}$ sodium phosphate $(\mathrm{pH} 8.0), 300 \mathrm{mM} \mathrm{NaCl}$, and $10 \mathrm{mM}$ imidazole (Lysis buffer), $50 \mathrm{mM}$ sodium phosphate (pH 8.0), $300 \mathrm{mM} \mathrm{NaCl}$, and $20 \mathrm{mM}$ imidazole (Wash buffer), $50 \mathrm{mM}$ sodium phosphate $(\mathrm{pH} 8.0), 300 \mathrm{mM} \mathrm{NaCl}$, and $250 \mathrm{mM}$ imidazole (Elution buffer), $50 \mathrm{mM}$ sodium phosphate (pH 7.4), 5\% glycerol and 0.05\% HECAMEG [methyl-6-O( $N$-heptylcarbamoyl)- $\alpha$-D-glucopyranoside] (Store buffer). Glycerol and HECAMEG are known to stabilize PHA synthase.

\footnotetext{
${ }^{1}$ Division of Biotechnology and Macromolecular Chemistry, Graduate School of Engineering, Hokkaido University, N13-W8, Kita-ku, Sapporo 060-8628, Japan

${ }^{2}$ Department of Biological Science and Technology, Tokyo University of Science, Yamazaki 2641, Noda 278-8510, Japan

*To whom correspondence should be addressed (Tel: +81-11-706-6612, Fax: +81-11-706-6612, E-mail: mken@eng.hokudai.ac.jp).
} 


\section{Purification of PHA Synthase}

E. coli strains JM109 (Takara, Japan) harboring pQEC1s were grown on $400 \mathrm{~mL}$ terrific broth medium at $20^{\circ} \mathrm{C}$ for $8 \mathrm{~h}$. Then, IPTG (isopropyl $\beta$-D-thiogalactopyranoside), final concentration $0.25 \mathrm{mM}$, was added into the flasks to induce the expression of PHA synthase gene. Cells were further cultured at $20^{\circ} \mathrm{C}$ for $16 \mathrm{~h}$. The harvested cells were rinsed with $400 \mathrm{~mL}$ ice-cold lysis buffer and resuspended in $50 \mathrm{~mL}$ ice-cold lysis buffer. The cells were disrupted by sonication. The crude extract was centrifuged at $15,000 \times g$ for $10 \mathrm{~min}$ and the supernatant was transferred into a new tube. Ni-NTA agarose beads (QIAGEN) suspension ( $5 \mathrm{~mL}$ each) was added to cellfree crude extract and the tube was incubated on ice with gentle mixing for $1 \mathrm{~h}$. The agarose beads were rinsed with $20 \mathrm{~mL}$ icecold wash buffer 5 times, and loaded on polypropylene columns (QIAGEN). The elution buffer $(2.5 \mathrm{~mL})$ was loaded on the column to elute PHA synthase. The eluted fraction was loaded on PD-10 columns (GE Healthcare), which was previously equilibrated by store buffer, and eluted by the addition of $3.5 \mathrm{~mL}$ store buffer to remove $\mathrm{NaCl}$ and imidazole. The purified PHA synthase was frozen in liquid nitrogen. The purified enzymes were stored at $-80^{\circ} \mathrm{C}$.

\section{Enzyme Activity Assay}

The activity of PHA synthase was determined by a modified spectroscopic assay based on the method described by Roo et $a l .{ }^{17}(R, S)$-3-hydroxybutyryl-CoA (3HB-CoA) and $(R S)-3$ hydroxyoctanoyl-CoA (3HO-CoA) were synthesized as described previously. ${ }^{14}$ Reaction mixture $(400 \mu \mathrm{L})$ contained $50 \mathrm{mM}$ sodium phosphate (pH 7.3), 50\% sucrose, $0.05 \%$ HECAMEG, $2 \mathrm{mM}$ DTNB [5,5'-dithiobis(2-nitrobenzoic acid)], $50 \mu \mathrm{g}$ PHA synthase and the substrates with various concentrations. After PHA synthase was added, absorbance at $412 \mathrm{~nm}$ was measured using spectrophotometer, which was homothermal at $30^{\circ} \mathrm{C}$. The kinetic parameters were determined based on one catalytic site in a dimmer enzyme.

\section{RESULTS AND DISCUSSION}

\section{Purification of PHA Synthase}

In vivo analysis results based on the composition of copolymers have provided useful insights for interpreting the mutational effects of PHA synthase on enzyme properties. Among beneficial mutants of PHA synthase, we chose three representative mutants for in vitro kinetic analysis. Substitution of glutamic acid (E) residue at position 130 with aspartic acid (D) (E130D mutation), and substitution of serine (S) residue at position 325 with threonine ( $\mathrm{T}$ ) (S325T mutation) increased SCL/MCL PHA content, but did not largely change its monomer composition (Table I). By contrast, substitution of glutamine (Q) at position 481 with lysine (K) (Q481K mutation) did not increase PHA content, but altered monomer composition. The combination of these beneficial mutations synergistically increased productivity and altered monomer composition of SCL/MCL copolymers.

Next, we purified wild-type PHA synthase and its mutants.
Table I. SCL/MCL PHA production in recombinant $E$. coli harboring mutated PHA synthases

\begin{tabular}{|c|c|c|c|c|}
\hline \multirow{2}{*}{ PHA synthase } & \multirow{2}{*}{$\begin{array}{l}\text { PHA content } \\
\text { (wt \%) }\end{array}$} & \multicolumn{2}{|c|}{$\begin{array}{c}\text { Monomer } \\
\text { composition (mol \%) }\end{array}$} & \multirow{2}{*}{ Ref. } \\
\hline & & $\begin{array}{l}\mathrm{SCL} \\
\left(\mathrm{C}_{4}\right)\end{array}$ & $\begin{array}{c}M C L \\
\left(C_{6-12}\right)\end{array}$ & \\
\hline Wild-type & 13 & 14 & 86 & 13 \\
\hline E130D & 20 & 17 & 83 & 13 \\
\hline S325T & 22 & 17 & 83 & 13 \\
\hline Q481K & 13 & 40 & 60 & 13 \\
\hline E130D/S325T & 17 & 13 & 87 & 13 \\
\hline E130D/Q481K & 32 & 42 & 58 & 13 \\
\hline S325T/Q481K & 28 & 31 & 69 & 13 \\
\hline E130D/S325T/Q481K & 9 & 28 & 72 & 13 \\
\hline
\end{tabular}

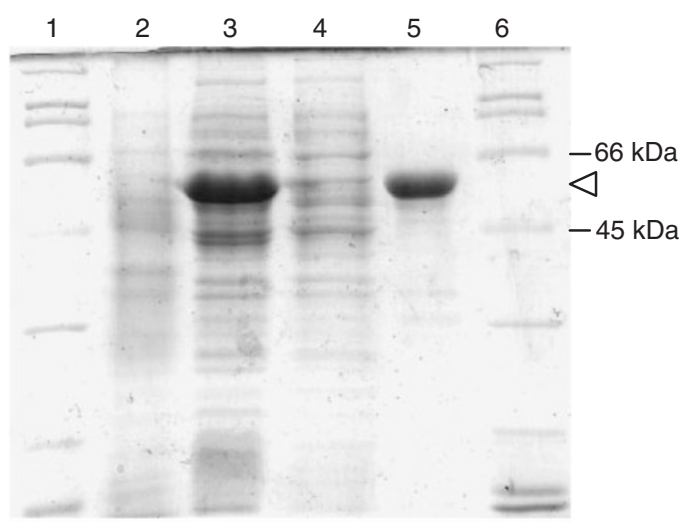

Figure 1. Purification of PHA synthase. White triangle indicates size of PHA synthase $(62 \mathrm{kDa}) .1,6$ : size marker, 2 : crude extract of $E$. coli harboring the control plasmid (pQE30), 3: crude extract of $E$. coli harboring PQEC1WT, 4: washed fraction, 5: eluted fraction.

The His-tag fusion of PHA synthase $(62 \mathrm{kDa})$ was highly accumulated as a soluble form in the recombinant $E$. coli, and purified by one step with a $\mathrm{Ni}^{2+}$ affinity column (Figure 1 ). The mutated PHA synthases were also purified as well as the wild-type enzyme (data not shown). The yields of the wild-type and mutated PHA synthases are listed in Table II. There were remarkable differences between the yields of enzyme. The E130D, S325T and especially pairwise E130D/S325T mutants were recovered with high yields, indicating that the E130D and S325T mutations increased the yield of PHA synthase. In contrast, the recovery of the $\mathrm{Q} 481 \mathrm{~K}$ mutant was very low $(10 \mathrm{mg} / \mathrm{L})$. Additionally the yields of E130D/Q481K and S325T/Q481K pairwise mutants were also lower than those of the E130D and S325T single mutants, respectively, indicating that the Q481K mutation negatively affected the yield of enzyme. This result was contrast to previous result that the accumulation level of Q481K mutant was similar to those of the wild-type and other mutated enzymes based on immunoblot analysis using the crude extracts from the recombinant E. coli. ${ }^{15}$ A major difference between previous and the present experiments was expression level of the enzymes. In the present study, PHA synthases were highly expressed using pQE vector that can be directly observed by SDS-PAGE (Figure 1), whereas in previous study, the expression levels were relatively 
Table II. Yield and activities of His-tag fusion of the wild-type and mutated PHA synthase from Pseudomonas sp. 61-3

\begin{tabular}{|c|c|c|c|c|c|c|c|}
\hline \multirow{2}{*}{$\begin{array}{l}\text { PHA } \\
\text { synthase }^{a}\end{array}$} & \multirow{2}{*}{$\begin{array}{l}\text { Yield } \\
(\mathrm{mg})^{\mathrm{b}}\end{array}$} & \multicolumn{3}{|c|}{ 3-hydroxybutyryl-CoA } & \multicolumn{3}{|c|}{ 3-hydroxyoctanoyl-CoA } \\
\hline & & $\begin{array}{c}k_{\mathrm{cat}} \\
\left(\mathrm{sec}^{-1}\right)\end{array}$ & $\begin{array}{c}K_{\mathrm{M}} \\
(\mathrm{mM})\end{array}$ & $k_{\text {cat }} / K_{\mathrm{M}}$ & $\begin{array}{c}k_{\mathrm{cat}} \\
\left(\mathrm{sec}^{-1}\right)\end{array}$ & $\begin{array}{c}K_{\mathrm{M}} \\
(\mathrm{mM})\end{array}$ & $k_{\text {cat }} / K_{\mathrm{M}}$ \\
\hline wild-type & 53 & $2.6 \times 10^{-2}$ & 1.6 & $1.6 \times 10^{-2}$ & 1.0 & 1.2 & 0.8 \\
\hline E130D & 83 & $6.6 \times 10^{-2}$ & 1.5 & $4.4 \times 10^{-2}$ & 2.4 & 1.4 & 1.7 \\
\hline S325T & 115 & $4.0 \times 10^{-1}$ & 30 & $1.3 \times 10^{-2}$ & 1.8 & 1.0 & 1.8 \\
\hline Q481K & 10 & $2.8 \times 10^{-2}$ & 4.4 & $6.0 \times 10^{-3}$ & 0.2 & 0.7 & 0.3 \\
\hline ED/ST & 135 & $1.4 \times 10^{-1}$ & 5.8 & $2.4 \times 10^{-2}$ & 7.3 & 4.3 & 1.7 \\
\hline ED/QK & 23 & $7.2 \times 10^{-1}$ & 2.1 & $3.4 \times 10^{-1}$ & 5.6 & 0.9 & 6.2 \\
\hline ST/QK & 45 & $4.6 \times 10^{-1}$ & 10 & $4.6 \times 10^{-2}$ & 6.3 & 4.7 & 1.3 \\
\hline ED/ST/QK & 60 & $8.6 \times 10^{-1}$ & 9.2 & $9.3 \times 10^{-2}$ & 11.4 & 6.4 & 1.8 \\
\hline
\end{tabular}

aED/ST: E130D/S325T, ED/QK: E130D/Q481K, ST/QK: S325T/Q481K, ED/ST/QK: E130D/S325T/Q481K. 'PProtein amount obtained from $1 \mathrm{~L}$ culture.

low that was detectable only by immunoblotting. From these facts, it was presumed that Q481K mutation decreased solubility of the enzyme, thus solution of the mutant with high concentration was hardly obtained.

\section{Assay of Enzymatic Activity}

The kinetic parameters of the wild-type and mutated PHA synthases were determined using two monomers, 3HB-CoA as a SCL monomer and 3HO-CoA as a MCL monomer (Table II). We chose $3 \mathrm{HO}-\mathrm{CoA}$ as a representative MCL monomer, because wild-type PHA synthase has highest activity toward 3HO-CoA. ${ }^{18}$ The value of $k_{\text {cat }}$ indicates the maximum turn over rate of enzyme. The value of $K_{\mathrm{M}}$ means concentration of substrate at which enzyme exhibits half reaction rate of the maximum, thus being an indicator of affinity to the substrate. The $k_{\text {cat }} / K_{\mathrm{M}}$ is used as an indicator of enzymatic activity. The E130D mutation increased $k_{\text {cat }}$ toward both substrates but $K_{\mathrm{M}}$ values were not significantly altered. In contrast, the S325T mutation greatly increased $k_{\text {cat }}$ and $K_{\mathrm{M}}$ for 3HB-CoA. The high $K_{\mathrm{M}}$ value means that the $\mathrm{S} 325 \mathrm{~T}$ mutation decreased affinity to 3HB-CoA. The results indicated that E130D and S325T mutants have different enzymatic properties, although these enzymes exhibited similar $\mathrm{P}(3 \mathrm{HB})$ and SCL/MCL PHA productions in vivo (Table I). The $\mathrm{Q} 481 \mathrm{~K}$ mutant exhibited lower $k_{\text {cat }} / K_{\mathrm{M}}$ toward $3 \mathrm{HO}-\mathrm{CoA}$ compared to the wild-type enzyme. This result was consistent with the fact that Q481K mutant accumulated less MCL fraction compared to the wild type (Table I).

The kinetic analysis showed that the wild-type enzyme exhibited comparable activity toward 3HB-CoA to some of the other mutants. This result was initially seemed to disagree with the fact that wild-type enzyme could produce only trace amount of $\mathrm{P}(3 \mathrm{HB})(0.1 \mathrm{wt} \%)$ in recombinant E. coli. ${ }^{15}$ However, the wild-type enzyme can produce SCL/MCL PHA containing significant amount of $3 \mathrm{HB}$ (Table I), indicating an intrinsic ability of the wild-type enzyme to incorporate $3 \mathrm{HB}$ moiety into the polymer. These phenomena could be interpreted by the effect of dimerization of PHA synthase. PHA synthases are known to form homodimer or heterodimer. The dimerization of subunits is a critical step in the initiation of polymerization. The wild-type enzyme might not be able to efficiently form a dimer without aid of MCL monomers, thus the enzyme hardly synthesizes $\mathrm{P}(3 \mathrm{HB})$. However, in the in vitro assay system, sucrose with high concentration, which promotes the dimerization of enzyme, ${ }^{19}$ was added to the reaction mixture that should facilitate the incorporation of 3HB-CoA.

The E130D/S325T pairwise mutant exhibited similar $k_{\text {cat }} / K_{\mathrm{M}}$ toward 3HO-CoA to the E130D and S325T single mutants. In fact, in vivo, the E130D/S325T mutant exhibited similar SCL/MCL PHA content than the single mutants (Table I). For activity toward 3HB-CoA, the E130D/S325T had intermediate properties between both single mutants. On the contrary, the combination of the E130D with Q481K mutation exhibited synergistic impact on the activity toward $3 \mathrm{HB}-\mathrm{CoA}$. The combination increased $k_{\text {cat }}$ for $3 \mathrm{HB}-\mathrm{CoA}$ but $K_{\mathrm{M}}$ was not largely increased. Thus, E130D/Q481K mutant has the highest $k_{\text {cat }} / K_{\mathrm{M}}$ for $3 \mathrm{HB}-\mathrm{CoA}$ among mutants tested. In addition, S325T/Q481K mutant has lower $K_{\mathrm{M}}$ compared to S325T mutant. The results suggested that the Q481K mutation combining with E130D and S325T improved binding affinity to the substrates.

In summary, the S325T mutation increased turn over of the enzyme, but led to a decrease in affinity to the substrates, whereas the E130D mutation enhanced activity without losing affinity. The Q481K mutant exhibited very weak activity. However, the Q481K mutation may contribute to the affinity to the substrate because of lowered $K_{\mathrm{M}}$ of multiple mutations with Q481K. In particular, the combination of the E130D with Q481K mutations greatly increased the activity and retained affinity to the substrates relatively high, indicating that the mutant should be suitable for the production of SCL/MCL PHA in the various hosts as well as E. coli.

\section{Correlation Between Yield of PHA Synthase and Molecular Weight of PHA}

The yielding amounts of purified PHA synthase, which were largely different depending on mutations (Table II), possibly reflect amount of soluble enzyme, because insoluble fraction was removed by centrifugation. The amount of soluble protein was supposed to influence the productivity of PHA within bacterial cells. However, the protein yields were not correlated to content of PHA in vivo (Table I and II). Instead, we found a correlation between the yields of enzyme and molecular weight 


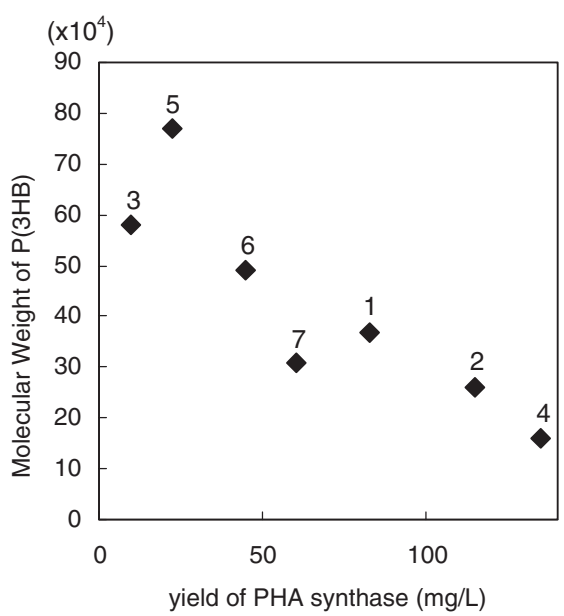

Figure 2. Correlation between yield of mutated PHA synthases and numberaverage molecular weight of $\mathrm{P}(3 \mathrm{HB})$ synthesized using the mutated enzymes. 1: E130D, 2: S325T, 3: Q481K, 4: E130D/ S325T, 5: E130D/Q481K, 6: S325T/Q481K, 7: E130D/S325T/ $\mathrm{Q} 481 \mathrm{~K}$. The molecular weight of $\mathrm{P}(3 \mathrm{HB})$ produced by the wild-type PHA synthase could not be determined because polymer content was too low.

of $\mathrm{P}(3 \mathrm{HB})^{14}$ previously synthesized using these mutated PHA synthases (Figure 2). To our knowledge, such correlation had not been reported for class II PHA synthases. However, for class I PHA synthase from Ralstonia eutropha, the activity and amount of enzyme are shown to influence the molecular weight of polymer. ${ }^{20,21}$ Additionally, a class III PHA synthase from Ectothiorhodospira shaposhnikovii, exhibited a good linear relationship between the polymer molecular weight and the monomer/synthase ratio by the in vitro polymer synthetic system. ${ }^{22}$ Therefore, although the mechanism determining molecular weight of PHA has not been revealed, the result in present study suggests that class I, II and III PHA synthases may have similar mechanism for regulating molecular weight of the polymer. For class II PHA synthases, the amount of soluble form rather than total amount of enzyme should influence the molecular weight. This is probably reason why the correlation of amount of enzyme and molecular weight had not been clear for class II PHA synthase in previous studies. The solubility of PHA synthase could be a factor causing the variation in molecular weight of PHAs produced by engineered PHA synthases. ${ }^{23}$

Acknowledgment. This work was partly supported by Grantin-aid for Scientific Research of Japan (Grants No. 16710059 to K.M., No. 18760587 to Y.S and No. 17360392 to S.T.) for the Ministry of Education, Culture, Sports, Science and Technol- ogy, Japan, Special Postdoctoral Research Program of RIKEN Institute (to K. M. and K. T.), Solution Oriented Research for Science, Technology (SORST) of the Japan Science and Technology Corporation (JST), Hokkaido Foundation for the Promotion of Scientific and Industrial Technology, and Industrial Technology Research Grant Program in 2003 from the New Energy and Industrial Technology Development Organization (NEDO).

Received: October 15, 2008

Accepted: December 8, 2008

Published: January 28, 2009

\section{REFERENCES}

1. T. Iwata, Y. Aoyagi, T. Tanaka, M. Fujita, A. Takeuchi, Y. Suzuki, and K. Uesugi, Macromolecules, 39, 5789 (2006).

2. D. Jendrossek, A. Schirmer, and H. G. Schlegel, Appl. Microbiol. Biotechnol., 46, 451 (1996).

3. Y. Doi, "Microbial Polyesters," Wiley-VCH, 1990.

4. L. L. Madison and G. W. Huisman, Microbiol. Mol. Biol. Rev., 63, 21 (1999).

5. S. Taguchi, H. Matsusaki, K. Matsumoto, K. Takase, K. Taguchi, and Y. Doi, Polym. Int., 51, 899 (2002).

6. H. Matsusaki, H. Abe, and Y. Doi, Biomacromolecules, 1, 17 (2000).

7. J. Stubbe and J. Tian, Nat. Prod. Rep., 20, 445 (2003).

8. A. Steinbüchel, K. Aerts, W. Babel, C. Föllner, M. Liebergesell, M. H. Madkour, F. Mayer, U. Pieper-Fürst, A. Pries, and H. E. Valentin, Can. J. Microbiol., 41, 94 (1995).

9. Y. Satoh, N. Minamoto, K. Tajima, and M. Munekata, J. Biosci. Bioeng., 94, 343 (2002).

10. H. Matsusaki, S. Manji, K. Taguchi, M. Kato, T. Fukui, and Y. Doi, J. Bacteriol., 180, 6459 (1998).

11. C. T. Nomura and S. Taguchi, Appl. Microbiol. Biotechnol., (2006).

12. S. Taguchi and Y. Doi, Macromol. Biosci., 4, 145 (2004).

13. S. Taguchi and T. Tsuge, in "Protein Engineering Handbook," S. Lutg and U. T. Boruscheuer, Ed., Wiley-VCH, 2008, p 877.

14. K. Matsumoto, K. Takase, E. Aoki, Y. Doi, and S. Taguchi, Biomacromolecules, 6, 99 (2005).

15. K. Takase, K. Matsumoto, S. Taguchi, and Y. Doi, Biomacromolecules, 5, 480 (2004).

16. K. Takase, S. Taguchi, and Y. Doi, J. Biochem., 133, 139 (2003).

17. G. de Roo, Q. Ren, B. Witholt, and B. Kessler, J. Microbiol. Methods, 41, 1 (2000).

18. K. Matsumoto, H. Matsusaki, K. Taguchi, M. Seki, and Y. Doi, Biomacromolecules, 3, 787 (2002).

19. S. Zhang, T. Yasuo, R. W. Lenz, and S. Goodwin, Biomacromolecules, 1, 244 (2000).

20. T. U. Gerngross and D. P. Martin, Proc. Natl. Acad. Sci. U.S.A., 92, 6279 (1995).

21. S. J. Sim, K. D. Snell, S. A. Hogan, J. Stubbe, C. Rha, and A. J. Sinskey, Nat. Biotechnol., 15, 63 (1997).

22. S. M. Zhang, S. Kolvek, S. Goodwin, and R. W. Lenz, Biomacromolecules, 5, 40 (2004).

23. T. Tsuge, S. Watanabe, S. Sato, T. Hiraishi, H. Abe, Y. Doi, and S. Taguchi, Macromol. Biosci., 7, 846 (2007). 